\title{
Factores de riesgo y protección asociados a la experiencia de ser madre: Un estudio comparativo entre México y los Estados Unidos*
}

\author{
Risk and protective factors associated with the experience \\ of being a mother: A comparative study between \\ Mexico and the United States of America
}

\section{Arturo Enrique Orozco Vargas* \\ ORCID: 0000-0002-2241-4234}

María del Rocío Mercado Monjardín ORCID: 0000-0002-9392-4100

Universidad Autónoma del Estado de México

Recibido: 7 de febrero de 2018 Revisado: 26 de marzo de 2018 Aceptado: 4 de mayo de 2018

\section{Resumen}

Ser madre es considerada una de las experiencias más significativas en la vida de una mujer. A pesar de su importancia en la sociedad, las mujeres mexicanas tienen que enfrentar múltiples desafíos dentro y fuera del hogar. El objetivo de este estudio fue conocer los efectos de la depresión, la violencia de pareja y los factores socioculturales presentes en México y los Estados Unidos en la experiencia de ser madre. El discurso de las participantes permitió identificar cinco temas eje: sus propias percepciones como madres, la influencia cultural, el involucramiento parental, los recursos que poseen y las barreras que enfrentan. La principal diferencia entre ambos grupos radica en el apoyo social que diversas organizaciones gubernamentales y no gubernamentales proporcionan a las madres inmigrantes en los Estados Unidos.

Palabras clave: madres mexicanas, inmigración, depresión, violencia de pareja, apoyo familiar y social.

Artículo de investigación. Como citar: Orozco, A. E. \& Mercado, M. R. (2019). Factores de riesgo y protección asociados a la experiencia de ser madre: Un estudio comparativo entre México y los Estados Unidos. Diversitas: Perspectivas en Psicología, 15(1), 71-85. DOI: https://doi. org/10.15332/s1794-9998.2019.0001.06

Correspondencia: Arturo Enrique Orozco Vargas dr.enrique.orozco@hotmail.com; María del Rocío Mercado Monjardín” rociomercado070@gmail.com 


\section{Abstract}

Being a mother is one of the most important experiences for many women. In spite of this fact, Mexican women have to face multiple factors affecting her motherhood. The purpose of this study was to examine the influence of depression, intimate partner violence as well as the sociocultural factors in Mexico and the United States in the experience of being a mother. In the narratives of these participants, we found five main themes: perceptions, cultural influence, involvement, resources, and barriers. The main difference between both groups was the social support that many governmental agencies and NGO's in the United States provide to immigrant mothers.

Keywords: mexican mothers, immigration, depression, intimate partner violence, family and social support.

\section{Introducción}

Alrededor del mundo y particularmente en Latinoamérica, el rol de madre ocupa un lugar prioritario en la sociedad (Baca Zinn, Eitzen \& Wells, 2014). Sin embargo, a pesar de la importancia de la maternidad y los múltiples roles que desempeñan las mujeres en el desarrollo integral de los hijos, en muchos países constituyen un grupo vulnerable debido a la influencia que ejercen los factores socioculturales, políticos y económicos (Organización de las Naciones Unidas [ONU], 2018). En términos generales, las mujeres latinoamericanas tienen un bajo nivel educativo, menos oportunidades de empleo y ascenso laboral, sus recursos económicos son más limitados, el acceso a los servicios públicos de salud es muy restringido y la mayoría de ellas tienen una doble labor dentro y fuera del hogar (Venguer, Fawcett, Vernon \& Pick, 1998). De la misma mane$\mathrm{ra}$, las mujeres latinas quienes han migrado a los Estados Unidos han reportado niveles más elevados de depresión, ansiedad, violencia de pareja y estrés así como discriminación, dificultades con el idioma, tensiones en el hogar y problemas económicos en comparación con mujeres pertenecientes a otros grupos étnicos (Alegría, Mulvaney-Day, Torres, Polo, Cao \& Canino, 2007; Dutton, Green, Kaltman, Roesch, Zeffiro, \& Krause, 2006).

En este contexto, múltiples elementos influyen en la experiencia de ser madre. Por una parte, factores de protección presentes en el contexto sociocultural, así como sus propias capacidades como madres que contribuyen positivamente en el desarrollo integral de sus hijos. Por ejemplo, la calidez materna es un factor determinante en la relación madre-hijo porque las madres quienes son cálidas y cariñosas con sus hijos, es más, tienden a establecer intercambios verbales y explicaciones apropiadas con respecto a los comportamientos deseables e indeseables que esperan ver en sus hijos (Esteinou, 2005). Asimismo, las mujeres quienes han desarrollado de manera satisfactoria las habilidades cognoscitivas que requieren para desempeñar su rol de madres reportan niveles más bajos de estrés parental (Tafarodi \& Swann, 1995). Por el contrario, diversos estudios llevados a cabo tanto en México como en los Estados Unidos han identificado una serie de factores los cuales disminuyen paulatinamente su desempeño y competencias como madres. (e.g., Arbona, Olvera, Rodriguez, Hagan, Linares \& Wiesner, 2010; Orozco, 2016; Terrazas-Carrillo, \& McWhirter, 2015). De manera particular, la depresión y la violencia de pareja son dos factores de riesgo que se han reportado como determinantes en el desempeño de los distintos roles que viven no solamente las madres quienes permanecen en sus países de origen, sino también aquellas quienes han decido emigrar a otros países.

Con respecto al impacto de la depresión, diversas investigaciones han examinado la relación entre los síntomas depresivos y ciertas variables entre las que 
destacan la falta de apoyo social y familiar (Da-Silva, Morales-Santos, Carvalho, Martins \& Teixeira, 1998), bajos niveles educativos (Morales-Carmona, LuqueCoqui \& Barroso-Aguirre, 2002), episodios previos de depresión (Ortega, Lartigue \& Figueroa, 2001) y la edad, situando a las mujeres menores de 40 años con los índices más altos de depresión (García de Alba, Castañeda, Pando \& Aranda, 2011).

Mientras que la prevalencia de la depresión en las mujeres mexicanas ha sido estimada entre el 5,8\% y el 19,8\% (Belló, Puentes-Rosas, Medina-Mora \& Lozano, 2005; Enríquez, González, Manríquez, Rivera, Pérez, \& Mendoza, 2010), estudios en Estados Unidos han documentado que la prevalencia de síntomas depresivos en las mujeres inmigrantes hispanas oscila entre el 8,4\% y el 38\% (Breslau \& Kendler, 2005; Masten et al., 2004). Debido a su condición de vulnerabilidad, los efectos de los síntomas depresivos en las mujeres inmigrantes tienen repercusiones aún más graves. Un estudio el cual incluyó la participación de inmigrantes latinas mostró que el estrés aculturativo, la falta de apoyo social, las expectativas con respecto al futuro, el bajo salario que reciben, el nivel educativo, las disfunciones familiares, la influencia de la religión, y el hecho de no encontrar oportunidades de crecimiento personal en sus países de origen estuvieron relacionados significativamente con la depresión (Hovey, 2000). De acuerdo con Bandura (1989), el desarrollo de las percepciones de auto-eficacia está ligado directamente con los estados emocionales que experimentan las personas. Esta noción ha sido comprobada a través de estudios empíricos los cuales han mostrado una relación negativa entre la depresión y los niveles de auto-eficacia materna (Weaver, Shaw, Dishion \& Wilson, 2008).

Con respecto a la violencia de pareja, los abusos físicos, psicológicos y sexuales que sufren las mujeres por parte de su pareja están relacionados directamente con el contexto sociocultural en el cual la violencia ocurre. Este contexto incluye factores tales como las normas sexistas, las instituciones patriarcales, la supremacía masculina, las tradiciones y las creencias étnicas las cuales son usadas por el perpetrador para condonar, apoyar y legitimar el uso de la violencia en contra de las mujeres (Harris, Firestone \& Vega, 2005). Diversos estudios han documentado repetidamente cómo los roles de género (Edelson, Hokoda \& RamosLira, 2007), el nivel educativo (Johnson, 2008), la violencia en la familia de origen (Rosenbaum \& Leisring, 2003), el estatus socioeconómico (Cunradi, Caetano \& Schafer, 2002), y el uso de las drogas y el alcohol (Flake \& Forste, 2006) están considerados entre los factores de riesgo más importantes en la prevalencia de la violencia de pareja en México y los Estados Unidos.

Aunque la violencia de pareja tiene consecuencias devastadoras en distintos aspectos de la vida de las mujeres, son muy escasos los estudios empíricos que han explorado los efectos de este tipo de violencia en el funcionamiento materno. Uno de estos estudios encontró que la violencia de pareja está asociada con conductas parentales controladoras, hostiles y rígidas, así como con niveles bajos de sensibilidad y apoyo parental (Gustafsson, Cox \& Blair, 2012). A su vez, otros estudios han revelado que la violencia de pareja está asociada con la falta de involucramiento parental y con formas de castigo verbal y físico por parte de los padres incluyendo golpes, gritos, nalgadas y amenazas (Krishnakumar \& Buehler, 2000). Asimismo, las mujeres quienes fueron abusadas por su pareja reportaron no solamente niveles más bajos de involucramiento y calidez en el trato con sus hijos, sino también mostraron comportamientos más agresivos, controladores y hostiles en la convivencia con sus hijos (Casanueva, Martin, Runyan, Barth \& Bradley, 2008).

Al revisar la literatura científica no se encontró ningún estudio que previamente haya analizado y comparado la influencia que tienen los síntomas depresivos, las relaciones maritales y las condiciones socioculturales en la experiencia de ser madre tanto en las mujeres inmigrantes de origen mexicano residiendo en los Estados Unidos como en las mujeres viviendo en México. Uno de los principales objetivos de este estudio fue llenar este vacío en la literatura, para lo cual, se analizó el discurso de las participantes con respecto a la depresión, la violencia de pareja y las percepciones que tienen acerca de sus capacidades como madres. De la misma manera, esta investigación comparó la influencia de los factores socioculturales presentes en México y 
los Estados Unidos y sus efectos en el desempeño materno de estos dos grupos de mujeres.

En este contexto, tres preguntas de investigación fueron formuladas: 1) ¿cuál es la influencia de la violencia de pareja y la depresión en sus capacidades como madres?; 2) ¿cuál es la descripción de las mujeres inmigrantes de origen mexicano residiendo en los Estados Unidos y las mujeres mexicanas con respecto a sus propias capacidades como madres?; 3) ¿cuál es el rol de los factores socioculturales presentes en México y los Estados Unidos y su impacto en el funcionamiento materno?

\section{Método}

\section{Participantes}

Dieciocho mujeres (10 en México y 8 en los Estados Unidos) participaron de manera voluntaria en este estudio ${ }^{1}$. Basados en la literatura científica y otros estudios empíricos, se diseñó una entrevista semi-estructurada con la finalidad de conocer sus experiencias como madres. El contenido de estas entrevistas tuvo como propósito investigar las percepciones de sus propias capacidades maternas, así como la influencia de la depresión y la violencia de pareja en estas percepciones. En el caso de las mujeres inmigrantes, se incluyeron además una serie de preguntas para examinar los beneficios y desafíos que tienen que enfrentar como madres al residir en un país extranjero. De las dieciocho madres quienes participaron, 15 de ellas estaban separadas. En cuanto al número de hijos que tenían, el rango osciló entre uno y cinco niños $(M=2,3$, $D E=1,15)$. El promedio de edad fue de 27,8 años $(D E=6,05)$. Con respecto al nivel educativo de las participantes, 8 madres tenían únicamente educación elemental, 4 de ellas terminaron la secundaria, 4 concluyeron la educación media superior y 2 madres tenían estudios de licenciatura.

$1 \quad$ Para diferenciar a estos dos grupos de participantes se nombrará madres mexicanas a aquellas viviendo en México y madres inmigrantes a quienes emigraron desde México y residen actualmente en los Estados Unidos.

\section{Procedimiento}

Las entrevistas tanto en México como en los Estados Unidos fueron hechas en español y tuvieron una duración de aproximadamente hora y media. Las madres en los Estados Unidos fueron contactadas a través del consulado mexicano. En esta institución se invitó a madres quienes hubieran sufrido violencia de pareja a participar en este estudio. Un total de 8 madres aceptaron participar en el estudio. Por otra parte, las participantes en México fueron contactas a través de agencias gubernamentales y organizaciones no gubernamentales (ONGs) las cuales proveen apoyo económico, legal y psicológico a las víctimas de la violencia de pareja. Con la ayuda de las autoridades de estas instituciones, 10 madres fueron invitadas a participar en este estudio. En ambos países las entrevistas fueron audio grabadas y transcritas en su totalidad. Un número de identificación fue asignado a cada caso para asegurar la confidencialidad y el anonimato de las participantes.

\section{Protocolo de entrevista}

La recolección de los datos se llevó a cabo mediante un cuestionario semi-estructurado el cual se diseñó ex profeso para este estudio. El protocolo incluyó preguntas relacionadas con el funcionamiento materno, la violencia de pareja, la depresión y los factores socioculturales presentes en México y los Estados Unidos.

\section{Método de análisis}

El presente estudio usó el método de comparación constante con la finalidad de analizar el discurso de las participantes. De acuerdo con Glaser (1965), el principal objetivo de este proceso reiterativo e inductivo es identificar y reducir la información obtenida a través de un análisis continuo. De esta manera, los investigadores no solamente son capaces de discernir las similitudes conceptuales y descubrir los principales patrones, sino también les es posible categorizar, codificar, delinear y conectar las categorías con el fin de desarrollar principios teóricos (Boeije, 2002). 
El método de comparación constante permitió un flujo continuo entre los datos recolectados y el análisis de estos con el objetivo de codificar la información en categorías. En la primera etapa se codificó cada rasgo presente en las entrevistas, comparándolo con otros rasgos previamente codificados en la misma categoría. En la segunda etapa se compararon los rasgos con las propiedades de las categorías. En este proceso se integraron todas las propiedades de cada categoría. La siguiente etapa estuvo caracterizada por el proceso de delimitar la teoría y la lista original de categorías. Con base en esta lista se comenzaron a integrar las propiedades de las categorías interrelacionadas. En la última etapa, después de haber codificado las narrativas de las participantes, el contenido de las entrevistas fue usado para desarrollar los 5 temas que emergieron. El análisis concluyó cuando los temas y categorías fueron depurados y se alcanzó la saturación de la información.

\section{Resultados}

El método de comparación constante permitió identificar las similitudes y diferencias en el discurso de las participantes. Cinco temas emergieron como resultado del análisis: percepciones, influencia cultural, involucramiento, recursos y barreras.

\section{Tema 1: Percepciones}

El primer tema que se identificó en las entrevistas fue el de las percepciones que las participantes tenían acerca de sí mismas. Dentro de este tema emergieron tres categorías. La primera de ellas está relacionada con sus propias percepciones como mujeres. Tanto las madres mexicanas como las inmigrantes se describieron a sí mismas como responsables, tenaces, competentes, honestas, inteligentes, espirituales, trabajadoras, respetuosas y disponibles para sus hijos. Una de las madres mexicanas comentó:

"Yo soy responsable, tranquila y una buena persona. Me preocupo y estoy al pendiente de mi hija, esposo, mi casa y las actividades escolares de mi hija" (Claudia², madre mexicana)

2 Los nombres que aparecen en este artículo fueron cambiados para proteger la identidad de las participantes.
Hablando acerca de su propia percepción como mujer, otra participante dijo:

"Yo soy una mujer muy trabajadora con muchas metas. Tengo sueños de superarme, no solamente en lo que se refiere al dinero, sino a estar estable. Me gustaría tener una familia estable. Yo trabajo muy duro, soy responsable y pienso que soy una buena madre" (Norma, madre inmigrante).

En la segunda categoría, las participantes hablaron de sus habilidades y capacidad como madres. Ambos grupos describieron su calidez materna, la preocupación por sus hijos, su habilidad para responder apropiadamente a las necesidades de sus hijos y el apoyo emocional que proveen a sus hijos como sus principales habilidades maternas.

Una madre lo describió de la siguiente manera:

"Yo me veo a mí misma como una madre amorosa. Para mí, lo más importante de una madre es su corazón. Pienso que yo guío a mi hijo con amor. Soy muy paciente y me gusta jugar con mis hijos. Sé cómo conseguir que mis hijos se interesen en lo que a ellos les gusta" (Marcela, madre mexicana).

Aunque las madres inmigrantes han estado viviendo en los Estados Unidos durante varios años, ellas mantienen un profundo contacto emocional y físico con sus hijos, el cual es muy característico en la cultura latina. Una madre describió este aspecto diciendo:

"Soy una madre muy amorosa. Soy estricta, pero al mismo tiempo trato de ser cariñosa. Una de mis habilidades como mamá es que yo hablo con mis hijas acerca de todo, mis sentimientos y el amor que tengo por ellas. Yo siempre les digo que las amo y que yo quiero lo mejor para ellas. Quiero sobretodo que sean exitosas en el futuro" (Sonia, madre inmigrante).

En el análisis de las responsabilidades maternas (tercera categoría) se identificaron la forma en que protegen a sus hijos y les proveen un ambiente cálido, conocen sus necesidades y promueven su desarrollo integral. Además de estar al pendiente 
de ellos en todo momento, las participantes viviendo en los Estados Unidos comentaron que tienen que conocer y seguir cuidadosamente las normas norteamericanas con respecto a la seguridad y cuidado de los niños. Por ejemplo, estas madres enfatizaron que cuando se ven en la necesidad de ir a trabajar por las tardes para ganar un ingreso extra, tienen que contratar a alguien quien se quede al cuidado de sus hijos mientras ellas están fuera del hogar. Una madre comentó este punto diciendo:

“Como le estaba platicando, es muy difícil ser una madre en un país extranjero porque tienes que buscar ayuda para todo. Aquí tienes que pagar para todo; si necesitas que alguien te cuide a tus hijos media hora para ir a la tienda o para que se quede cuidándolos cuando no tienen clases mientras estás trabajando, necesitas pagarle a alguien para que se esté con ellos. Si yo estuviera en México sería más fácil porque mi mamá me podría ayudar con eso [cuidar a los hijos]" (Julia, madre inmigrante).

\section{Tema 2: Influencia cultural y familiar}

El segundo tema que emergió de las entrevistas fue la influencia de la cultura. La primera categoría dentro de este tema fue el sistema familiar. Cuando la mayoría de las participantes describieron la influencia de las personas o las instituciones en su rol como madres, ellas mencionaron que su propia madre fue un modelo muy importante. Las participantes dijeron que ellas aprendieron de sus madres cómo satisfacer las necesidades básicas de sus hijos, darles el apoyo emocional que necesitan, ayudarlos en sus actividades escolares y protegerlos. Una participante comentó acerca de su propia madre lo siguiente:

"Mi experiencia más grande fue ver cómo mi mamá se desvivió por nosotros. De mi mamá yo aprendí que los hijos son lo primero y después ya puedo pensar en mí. Recuerdo que mi mamá tuvo que trabajar para sacarnos adelante y que no nos faltara lo indispensable porque el salario de mi papá era muy bajo" (Ana, madre mexicana).
Además de describir la importancia de su propia madre como un modelo para su labor materna, las participantes describieron los métodos de disciplina que ellas emplean con sus hijos (segunda categoría). Fue muy interesante que la mayoría de las participantes mencionaran que ellas no usan los mismos métodos de disciplina violentos que sus padres sí usaron. Las participantes narraron cómo era muy frecuente que sus padres las golpearan o les dieran nalgadas para corregir su comportamiento. Sin embargo, ambos grupos de madres han aprendido que éste no es el mejor método. Ellas prefieren hablar con sus hijos y explicarles lo que ellas consideran es un comportamiento adecuado. Aunque fue muy común para sus padres usar técnicas de disciplina punitivas, las participantes mencionaron que cuando ellas hablan con sus hijos, obtienen mejores resultados.

Una tercera categoría que surgió de las narrativas fue la importancia de la familia nuclear. En su discurso, la mayoría de las madres enfatizaron que una de las metas más trascendentales en su vida era mantener a su pareja y sus hijos viviendo en un hogar estable, cálido y perdurable. Esta fue una de las principales razones que tuvieron para soportar todo tipo de abuso y con ello evitar a toda costa el divorcio o la separación. Las participantes también mencionaron que vivir en una familia tradicional había sido desde el comienzo de su matrimonio una de sus prioridades. Durante el tiempo que vivieron con su pareja, hicieron muchos sacrificios, aguantaron todo tipo de malos tratos y buscaron reconciliar sus diferencias con el propósito de mantener unida a su familia. Una de ellas lo explicó de la siguiente manera:

“Desde que yo vivía en México, yo aprendí que la familia es lo más importante para una madre y sus hijos. Al principio yo no aceptaba esta idea [la de divorciarse] porque soñaba con una familia donde todo sería maravilloso. Fue muy difícil porque pensaba que la mejor herencia que les podía dejar a mis hijos era una familia unida y cercana" (Yolanda, madre inmigrante).

Las participantes mexicanas como las inmigrantes describieron la manera en que los valores y las tradiciones son muy importantes para ellas (cuarta 
categoría). Todas las madres mencionaron algunos valores que tratan de inculcar a sus hijos. Señalaron principalmente la honestidad, el respeto, la tolerancia, la puntualidad y la responsabilidad como valores que continuamente les están enseñando a sus hijos. Una de las participantes enfatizó que los padres deben proveer una educación basada en valores y no solamente limitarse a los conocimientos que adquieren en la escuela. Ella comentó:

"Es muy importante que los padres enseñen a sus hijos los valores porque una cosa es la educación académica y algo distinto es la educación que los niños reciben en sus casas. Yo he conocido personas muy exitosas, pero que no tienen valores. Estas personas dicen algo y hacen todo lo contrario" (Marcela, madre mexicana).

Finalmente, la quinta categoría en este tema estuvo relacionada con las creencias presentes en la cultura mexicana y la influencia que tienen en su rol como madres. Por ejemplo, las participantes mexicanas piensan que una madre debe estar al pendiente de sus hijos, proveerles comida, estar involucrada en las actividades escolares de sus hijos y protegerlos. Por otra parte, aunque las madres entrevistadas en los Estados Unidos han estado en contacto directo con la cultura norteamericana, es muy interesante observar que ellas mantienen las mismas creencias, valores, tradiciones y costumbres que las madres viviendo en México. Una de ellas comentó a este respecto lo siguiente:

“Desde que me vine a los Estados Unidos, mi rol como madre no ha cambiado porque yo conservo mis raíces. Yo no voy a vivir como los americanos o como los afroamericanos. Siempre tendré mis raíces bien firmes porque la cosa más importante para alguien son sus valores. Por ejemplo, la Navidad aquí es muy diferente, pero no le hace y nosotros celebramos la Navidad como si estuviéramos en México" (Mayra, madre inmigrante).

De la misma manera, aunque las madres inmigrantes han tenido la oportunidad de establecer un contacto con las madres norteamericanas o las madres de otros grupos minoritarios, ellas prefieren interactuar con otras madres mexicanas o hispanas. En su discurso, todas mencionaron que son capaces de entender y expresar algunas ideas en inglés; sin embargo, tienen muchas dificultades para hablar con fluidez. Esta es la principal razón por la cual prefieren participar en programas que se ofrecen en español, hablar con las maestras de sus hijos en español y convivir con madres hispanas en su lengua nativa. Por ende, su rol como madres es influenciado por creencias, comentarios, conversaciones formales e informales, consejos e ideas provenientes de su misma cultura.

\section{Tema 3: Involucramiento}

El tercer tema que surgió de las entrevistas fue codificado como involucramiento. La primera categoría relacionada con este tema fue la manera en que las madres responden a las necesidades básicas de sus hijos. De manera particular, las madres mexicanas narraron todo lo que ellas hacen para que sus hijos tengan un desarrollo físico, cognoscitivo, social y emocional adecuado. Llamó la atención que su discurso estuvo lleno de múltiples ejemplos a través de los cuales dieron cuenta de todos los esfuerzos que diariamente hacen para establecer una relación armónica y satisfactoria con sus hijos. Aunque tienen que enfrentar una condición económica precaria y dedican muchas horas a trabajar fuera del hogar, estas madres tienen la tenacidad para superar diversos obstáculos como lo son la falta de apoyo, los conflictos maritales y el estrés. Para ellas, su principal motivación para salir adelante son sus hijos y están dispuestas a hacer todo lo que esté en sus manos para darles una mejor vida. Una de ellas describió sus esfuerzos en estos aspectos:

"Yo siempre la llevo a sus vacunas. Cuando se enferma, la llevo al doctor. En las tardes le ayudo con sus tareas y le reviso lo que hizo durante el día [en su escuela] para saber cómo le está yendo. Regularmente voy a hablar con su maestra para saber cómo va en la escuela. Me gusta estar al pendiente de todo lo que ella hace en la escuela" (Alba, madre mexicana).

Todas las madres inmigrantes también hablaron acerca de lo que hacen para proveer un ambiente estimulante y cálido. Las madres inmigrantes se enfocaron en proveer un apoyo constante en la salud, la nutrición, el desempeño académico y 
las interacciones sociales de sus hijos. El siguiente ejemplo nos habla de esto:

"Yo siempre hablo con sus maestras para estar enterada del progreso de mi hijo y cuando ellas necesitan hablar conmigo me mandan llamar. Yo les he comentado de la situación que estamos viviendo en estos momentos en la casa, así que ellas están enteradas de todo" (Alma, madre inmigrante).

La segunda categoría incluida en este tema fue la sensibilidad. En la cultura mexicana, el amor, el afecto, la calidez y el cariño son elementos decisivos para muchas madres. A lo largo de las entrevistas, todas las madres mexicanas mencionaron varias veces cómo su maternidad está basada en el amor que ellas les dan a sus hijos. Fue muy interesante conocer que cuando ellas no tienen suficientes recursos económicos, las participantes mexicanas consideran que el amor es su principal recurso como madres. Una de estas madres comentó lo siguiente:

“Para mí es muy importante el contacto físico que pueda tener con mi hija. Yo baso mi rol como madre en el amor que le doy. Yo he criado a mi hija con amor, evitando imposiciones porque respeto lo que ella quiere. Yo pienso que el amor es la base de todo" (Claudia, madre mexicana).

La última categoría que se incluyó en este tema fue la interacción madre-hijo. Aunque todas las madres inmigrantes han vivido en los Estados Unidos por lo menos 10 años, las interacciones que establecen con sus hijos son muy similares a aquellas descritas por las madres en México. Estas interacciones están caracterizadas por el tiempo, en cantidad y calidad, que pasan con sus hijos. La mayoría de las madres inmigrantes mencionaron que tratan de pasar la mayor parte de su tiempo libre conviviendo con sus hijos. En vez de dedicarse a realizar sus actividades personales, descansar más tiempo o visitar a sus amistades, ellas prefieren estar en la casa viendo películas, comiendo o jugando con sus hijos. Cuando tienen dinero, llevan a sus hijos a algún parque, al cine $o$ a un sitio de entretenimiento infantil y cuando carecen de los recursos económicos necesarios, juegan con sus hijos en un parque público.

\section{Tema 4: Apoyo}

En los 3 temas anteriores fue posible identificar una gran cantidad de elementos comunes entre las madres mexicanas y las madres inmigrantes. Sin embargo, en este tema el discurso de las participantes describe muy claramente las diferencias en el apoyo que recibe cada grupo. En México, las familias de escasos recursos económicos enfrentan una serie de desafíos para satisfacer sus necesidades básicas. Esta situación es incluso peor para las mujeres quienes son madres solteras o quienes están divorciadas porque ellas tienen que hacerse cargo de todos los gastos de la casa. Dentro de su precariedad económica, las madres mexicanas enfatizaron el apoyo que reciben de su familia especialmente cuando sus padres les ayudan con el cuidado de sus hijos. En su discurso, siete madres mexicanas hablaron acerca de cómo recurren a sus padres cuando necesitan quedarse horas extra en su lugar de trabajo o se les presenta un evento inesperado al que deben asistir.

"Mi mamá me ayuda a cuidar de mi hija durante las tardes. Ella es quien le da de comer y le ayuda a hacer la tarea. Cuando yo paso por ella a las 10 de la noche, ella ya me la entrega con la pijama lista para dormir" (Citlalli, madre mexicana).

Además del apoyo familiar que reciben estas participantes, solamente tres de ellas reciben una pequeña cantidad de dinero que el gobierno les otorga. En consecuencia, las madres mexicanas quienes no tienen familiares viviendo cerca de ellas para ayudarles con sus hijos enfrentan un sinfín de dificultades para cumplir con sus responsabilidades como madres.

Por el contrario, las madres inmigrantes tienen un escenario radicalmente diferente. Aunque la mayoría de las participantes en este estudio son indocumentadas, ellas reciben diversos apoyos tanto de agencias gubernamentales como de organizaciones civiles. Como resultado de esto, estas madres tienen la posibilidad de superar la mayoría de los obstáculos que los inmigrantes encuentran regularmente en su proceso de aculturación. Uno de los principales apoyos que reciben proviene de la policía. Después de haber sido violentadas por su 
pareja, este grupo de participantes reportaron el abuso a la policía y pidieron que se les protegiera a ellas y a sus hijos. Una de ellas describió esta ayuda de la siguiente manera:

"Cuando fui abusada por mi esposo, recibí una orden de restricción por tres años. La policía me ayudó pues mi esposo fue arrestado y más tarde lo metieron a la cárcel. Esa fue la única forma en que las cosas se calmaron porque la situación ya era para mí insoportable" (Norma, madre inmigrante).

El hecho de haber sido víctimas de violencia de pareja les ha traído paradójicamente varios beneficios. Por ejemplo, la protección de la policía, así como la asesoría legal que han recibido de distintas organizaciones no gubernamentales ha sido determinante para conseguir la custodia absoluta de sus hijos y comenzar con el proceso de divorcio. Además de esto, este grupo de participantes han recibido terapia individual y grupal a través de la cual han recuperado su estabilidad emocional. Estos beneficios los ilustraron de la siguiente manera:

"Yo he tenido también acceso a abogados de manera gratuita. Yo no sabía que hay abogados que ayudan a las mujeres que han sufrido violencia sin cobrarles un solo dólar. En los Estados Unidos muchas mujeres no saben de esto y por esa razón hay tantas que son abusadas por su marido. Desafortunadamente ellas no pueden escapar de esta situación porque no saben que hay todas estas organizaciones que les pueden ayudar" (Rosana, madre inmigrante).

Otro de los apoyos más importantes para ellas lo han encontrado en los programas para padres. Como se mencionó anteriormente, debido a la escasez de estos programas en México, las participantes mexicanas basan su desempeño como madres en su instinto materno. Por el contrario, las madres inmigrantes tienen la oportunidad de participar en varios programas para padres, especialmente aquellos que se imparten en las escuelas de sus hijos. Estas madres recibieron clases a través de las cuales aprendieron diversas estrategias para realizar una correcta crianza de sus hijos, establecer métodos de disciplina eficaces y crear un ambiente familiar armónico.
"Fue muy útil para mí participar en los programas para madres porque las instructoras están siempre enseñándonos y ofreciéndonos clases para llegar a ser unas buenas mamás. Yo aprendí cómo tratar de mejor manera a mis hijos. Ellas siempre están tratando de ayudar a las mamás. En estos programas yo he aprendido las cosas más útiles" (Mayra, madre inmigrante).

\section{Tema 5: Barreras}

La experiencia de ser madre es a menudo difícil y desafiante. Cuatro categorías ilustraron los obstáculos más comunes a los que las madres mexicanas e inmigrantes se enfrentan todos los días. La primera de ellas describió los conflictos maritales como una barrera. Como se señaló anteriormente, la violencia de pareja tiene distintos efectos para las madres mexicanas y para las madres inmigrantes. En lo particular, las madres mexicanas quienes han sido víctimas de violencia doméstica además de presentar ansiedad, baja autoestima, depresión, conductas antisociales, ideas suicidas, miedos, dificultades para dormir y desconfianza en las personas, tienen que salir a trabajar para satisfacer las necesidades básicas de su familia. A esto hay que añadir que el apoyo que reciben de instancias gubernamentales es mínimo. Ellas solamente hablaron de la terapia que reciben ocasionalmente y los consejos que les brindaron al presentar su denuncia. Ninguna de ellas mencionó algún tipo de asesoramiento o seguimiento que se le está dando a su situación. Una de las participantes mexicanas ilustró los efectos negativos que la violencia de pareja ha generado en su desempeño como madre:

"Cuando tuve estos problemas con mi esposo, dejé de ponerle atención a las actividades escolares de mi hija. Algunas veces mi mamá me decía que la maestra [de mi hija] le había pedido una tarea especial y yo no quería saber nada de eso. Otras veces me excusaba diciendo que estaba muy ocupada y que no tenía tiempo de ayudar a mi hija" (Magdalena, madre mexicana).

La misma situación experimentaron las participantes residiendo en los Estados Unidos quienes sufrieron todo tipo de abusos afectando directamente su 
desempeño como madres. Una de ellas describió esta situación de la siguiente manera:

"Cuando tuve problemas con mi esposo, la relación con mi hijo se vio afectada porque todo el tiempo estaba triste. Mi esposo siempre me decía que todos los problemas que teníamos eran por mi culpa. Si los niños no iban bien en la escuela me echaba la culpa. Con todos esos sentimientos encima de mí, yo no tenía ganas de hacer absolutamente nada" (Norma, madre inmigrante).

La segunda categoría que se identificó está relacionada con las consecuencias de la depresión. Las madres mexicanas mencionaron que la depresión tiene efectos negativos en su rol como madres. Por ejemplo, un factor muy común en ellas fue la falta de entusiasmo para planear sus actividades cotidianas. La mayoría de ellas lo único que deseaban era estar solas evitando en lo posible el contacto con amistades y familiares. Como consecuencia de esta situación, la mayoría de ellas dejaron de prestar atención a sus hijos. A su vez, limitaron sus responsabilidades como madres al dejar de cocinar, limpiar la casa y estar atentas al comportamiento de sus hijos en el hogar. Una de ellas mencionó:

"Cuando estaba deprimida, no ponía atención a nada. No cuidé de mi hija de la misma manera que lo hacía cuando me sentía bien. Cuando estaba deprimida, me sentía desconectada de mis responsabilidades. Ignoraba muchas cosas y lo peor es que yo me daba cuenta de eso. Reconozco que no hice lo que yo debía de hacer como madre" (Leonor, madre mexicana).

De la misma manera, una madre inmigrante describió efectos similares:

"La depresión tuvo muchas consecuencias negativas para mi vida. Tuve muchas crisis emocionales las cuales hacían que perdiera la cabeza. Hacían que me volviera loca. No quería que mis hijos estuvieran cerca de mí. Viví con mucho estrés y sobre todo me faltó el apoyo de mi esposo. No quería que nadie me viera, dejé de visitar a mi familia y me sentía muy triste. Recuerdo pasarme todo el día llorando y sentirme frustrada conmigo misma" (Alma, madre inmigrante).
La tercera categoría que expresaron las madres inmigrantes está relacionada con su estatus migratorio y los desafíos propios del proceso de aculturación. El mayor obstáculo para ellas ha sido la falta de fluidez en el idioma inglés. El hecho de no hablar inglés ha dificultado enormemente su adaptación a la cultura norteamericana. Por ejemplo, no han podido continuar con sus estudios y por ende son incapaces de conseguir un mejor trabajo porque no tienen fluidez en el idioma inglés. Incluso las actividades cotidianas como lo es ir al doctor, pagar los servicios del hogar, hablar con las maestras de sus hijos y comprar alimentos en las tiendas resulta una tarea muy difícil. A este respecto una de ellas comentó:

"Ser madre [en un país extranjero] es muy difícil cuando no se sabe el idioma. Es muy frustrante cuando no hablas inglés porque la gente me habla de muchas cosas y yo solamente puedo entender tres o cuatro palabras. En cualquier país extranjero, el principal desafío es el idioma. La barrera del idioma es lo que hace las cosas más difíciles. En la escuela de mis hijas puedo entender algunas cosas, pero cuando trato de expresar mis preocupaciones tengo muchos problemas para decir lo que quiero" (Isabel, madre inmigrante).

Todas las madres que tomaron parte en este estudio inmigraron de forma ilegal a los Estados Unidos; por consiguiente, ninguna tiene documentos para trabajar. Al no contar con un permiso oficial para trabajar, no tienen la oportunidad de recibir los beneficios que obtienen las inmigrantes documentadas. Esta situación las ha acompañado durante toda su estadía en los Estados Unidos. Debido a esta condición, este grupo de madres solamente ha podido trabajar limpiando casas y edificios públicos, trabajando en la cocina de los restaurantes o desempeñando labores como obreras. Aunado a esto se encuentra siempre presente el miedo de ser deportadas. El solo hecho de pensar que un día pueden ser arrestadas por la policía y las separen de sus hijos crea un ellas altos niveles de estrés y angustia.

Finalmente, otra de las principales barreras está relacionada con los recursos económicos que poseen. La mayoría de las participantes mencionaron que su 
situación económica es sumamente precaria. Estas madres trabajan jornadas muy largas y cuando no tienen suficiente dinero deben cubrir dos turnos. Estas familias tienen dinero únicamente para satisfacer sus necesidades básicas y sus posibilidades para mejorar su condición económica son casi nulas. Una de estas madres comentó:

"Algunas veces no puedo manejar los problemas en mi trabajo y mi situación económica es muy difícil. Cuando me siento aplastada por la presión económica, soy más intolerante y distante con mi hija. Mi mayor problema es conseguir el dinero para pagar todos los gastos de la casa y eso es demasiado para mí sola" (Yoselin, madre mexicana).

Por el contrario, la mayoría de los inmigrantes llegan a los Estados Unidos con el propósito de encontrar un buen trabajo para ofrecerles un mejor futuro a sus hijos. Sin embargo, su situación económica es frecuentemente muy inestable. En su discurso, este grupo mencionó que es difícil conseguir un trabajo donde paguen un buen salario porque no tienen estudios superiores y su nivel de inglés es deficiente. Como resultado de esto, la mayoría de las inmigrantes se dedican a limpiar casas o edificios y otras trabajan en la cocina de los restaurantes.

"Ser madre aquí [en los Estados Unidos] es muy difícil porque las madres americanas hablan inglés y han tenido la oportunidad de estudiar. Otro de los principales obstáculos que tengo es mi situación económica porque si yo ganara más dinero tendría la oportunidad de pasar más tiempo con mis hijos y esto me haría una mejor mamá" (Yolanda, madre inmigrante).

\section{Discusión}

Debido a la escasez de investigaciones relacionadas con la experiencia de ser madre, el objetivo de este estudio cualitativo fue investigar la influencia que tiene el contexto sociocultural, la violencia de pareja y la depresión en el desempeño de los roles maternos de las mujeres mexicanas y aquellas quienes han inmigrado a los Estados Unidos. El discurso de ambos grupos de madres permitió un mayor entendimiento de los múltiples factores pre- sentes en la cultura mexicana y norteamericana los cuales no siempre son considerados en los estudios cuantitativos.

Los resultados encontrados confirmaron los principios teóricos postulados por Bandura con respecto a la influencia que tienen distintos factores en la conformación de las percepciones que las personas tienen de sus propias capacidades. A pesar de vivir en dos contextos muy diferentes, uno de los principales hallazgos de la presente investigación reveló que las madres mexicanas y las madres inmigrantes comparten los mismos valores y creencias con respecto a la maternidad. Aunque las madres inmigrantes han vivido en los Estados Unidos por lo menos una década, este grupo conserva las mismas tradiciones, costumbres, creencias y prácticas parentales que sus similares en México. De la misma manera, las madres inmigrantes desempeñan sus actividades cotidianas dentro y fuera del hogar como si ellas estuvieran viviendo en México. Por ejemplo, todos los días cocinan comida mexicana, asisten a los servicios religiosos en español, compran sus enseres y alimentos en supermercados mexicanos, ven programas de televisión solamente en español, cuando tienen oportunidad comen en restaurantes mexicanos, mantienen un contacto muy cercano con otras madres mexicanas e hispanas y participan en eventos para latinos. Este conjunto de patrones son los que explicaría por qué no se encontraron indicios de la influencia que la cultura norteamericana tiene en las percepciones de sus capacidades como madres.

La influencia de la violencia de pareja en sus capacidades como madres fue uno de los objetivos de la presente investigación. Contrario a las similitudes encontradas en la mayoría de las otras categorías, el análisis del discurso de las participantes reveló importantes diferencias con respecto a los efectos de la violencia de pareja. La principal diferencia está relacionada directamente con la protección, la orientación y la asistencia gratuita que reciben las víctimas residiendo en los Estados Unidos. Como se ha señalado, diversas investigaciones han documentado que las mujeres inmigrantes provenientes de Latinoamérica representan uno de los grupos étnicos más vulnerable y marginalizado en los Estados Unidos (e.g., Cavazos-Rehg, Zayas \& Spitznagel, 
2007; Parrado \& Flippen, 2005; Sullivan \& Rehm, 2005). Sin embargo, no hay estudios analizando la influencia de los factores de protección en la violencia de pareja. El presente estudio llenó este vacío en la literatura e identificó de manera precisa algunos aspectos relacionados con la importancia que los recursos sociales, familiares, legales y económicos tienen para las mujeres inmigrantes quienes han sido abusadas por su pareja.

En su discurso, todas las madres inmigrantes señalaron los diversos beneficios que pueden obtener por parte de las organizaciones gubernamentales y las ONG's. En los Estados Unidos, cuando una mujer es abusada por su pareja, sin importar su estatus migratorio, puede recibir protección, ayuda y una gran variedad de apoyos. De la misma manera, este grupo de mujeres recibió ayuda legal para continuar con el proceso de divorcio y obtener más tarde la custodia legal de sus hijos. Como resultado de esto, se sienten más seguras y convencidas que tomaron la decisión correcta al abandonar a su pareja y comenzar una nueva vida al lado de sus hijos. Asimismo, las madres inmigrantes recibieron apoyo emocional proporcionado por instituciones no gubernamentales las cuales les brindaron la posibilidad de participar en grupos terapéuticos, programas de auto-ayuda y talleres. Este apoyo fue determinante para mitigar en gran medida los obstáculos propios del proceso de inmigración y al mismo tiempo explica las diferencias tan marcadas entre ambos grupos de participantes con respecto a la influencia de la violencia de pareja en su desempeño como madres.

Además de investigar los efectos de la violencia de pareja, otro de los objetivos de este estudio fue examinar el impacto de la depresión en sus capacidades como madres. Como se señaló anteriormente, la depresión es uno de los trastornos mentales más comunes en esta población. Los síntomas depresivos tienen efectos directos en distintas áreas del desarrollo humano siendo el estado emocional una de ellas. El análisis cualitativo que se llevó a cabo permitió determinar el impacto que la depresión tiene en las percepciones de ambos grupos de participantes. Sin ninguna excepción, todas las madres mencionaron los distintos síntomas depresivos que experimentaron entre los que se incluyen la tristeza, apatía, frustración, indiferencia, pérdida de confianza, desaliento, culpa, vergüenza y desesperanza.

Además de estos síntomas, algunas de ellas expresaron que comenzaron a evitar la convivencia con sus amigos y los miembros de su familia. Estos sentimientos tuvieron efectos negativos no solo para su estabilidad emocional, sino también para su efectividad como madres al decrecer significativamente el contacto con sus hijos. Una segunda consecuencia de esta condición fue el desinterés por superarse. Como resultado de esto, las participantes limitaron su vinculación en actividades que podrían haber mejorado sus capacidades maternas. Finalmente, tanto las madres inmigrantes como las mexicanas admitieron que cuando experimentaron todos estos sentimientos, la atención que les brindaron a sus hijos dentro del hogar así como en lo relacionado con sus actividades escolares decayó drásticamente.

Con respecto al rol de los factores socioculturales presente en México y los Estados Unidos, los resultados de esta investigación permitieron identificar con precisión la importancia que tiene el apoyo social en la vida de las inmigrantes. Aunque algunos estudios han documentado que ser madre en un país extranjero es más difícil (e.g., Bacallao \& Smokowski, 2007; Parra-Cardona, Bulock, Imig, Villaruel, \& Gold, 2006), el discurso de las madres que participaron en este estudio reveló lo contrario. A pesar de ser indocumentadas y enfrentar todos los días distintos obstáculos, cada una de las madres inmigrantes dio cuenta de los múltiples beneficios que no solamente ellas, sino especialmente sus hijos han recibido. De manera particular, por el hecho de ser ciudadanos norteamericanos, sus hijos reciben servicios médicos y escolares de forma gratuita, ayuda financiera para cubrir los pagos relacionados con la vivienda y la alimentación, así como la oportunidad de participar en actividades recreativas. Entre los principales beneficios que sus hijos han obtenido en los Estados Unidos destaca la educación. Para estas participantes, uno de los motivos para emigrar hacia los Estados Unidos fue el pensar que sus hijos encontrarían en este país mejores oportunidades académicas. En su discurso, todas las madres inmigrantes coincidieron al señalar 
que todos los sacrificios han valido la pena con tal de ofrecerles un futuro más promisorio a sus hijos.

Los principales hallazgos del presente estudio cualitativo permitieron conocer a profundidad no solamente las alegrías y las tristezas, las satisfacciones y las frustraciones, los logros y los retos a los que día a día se enfrentan las mujeres mexicanas en ambos lados de la frontera norte, sino al mismo tiempo las múltiples expresiones de coraje, perseverancia, dedicación y de manera muy particular, el profundo amor que tienen por sus hijos.

\section{Referencias}

Alegría, M., Mulvaney-Day, N., Torres, M., Polo, A., Cao, Z., \& Canino, G. (2007). Prevalence of psychiatric disorders across Latino subgroups in the United States. American Journal of Public Health, 97(1), 68-75. Doi: http://doi. org/10.2105/AJPH.2006.087205

Arbona, C., Olvera, N., Rodriguez, N., Hagan, J., Linares, A., \& Wiesner, M. (2010). Acculturative stress among documented and undocumented Latino immigrants in the United States. Hispanic Journal of Behavioral Sciences, 32(3), 362-384. Doi: http://doi.org/10.1177/0739986310373210

Baca Zinn, M., Eitzen, D. S., \& Wells, B. (2005). Diversity in families. Boston: Allyn and Bacon.

Bacallao, M. L. \& Smokowski, P. R. (2007). The costs of getting ahead: Mexican family system changes after immigration. Family Relations, 56(1), 52-66. Doi: http://doi. org/10.1111/j.1741-3729.2007.00439.x

Bandura, A. (1989). Regulation of cognitive processes through perceived self-efficacy. Developmental Psychology, 25(5), 729-735.

Belló, M., Puentes-Rosas, E., Medina-Mora, M. E., \& Lozano, R. (2005). Prevalencia y diagnóstico de depresión en población adulta en México. Salud Pública de México, 47(1), 4-11.

Boeije, H. (2002). A purposeful approach to the constant comparative method in the analysis of qualitative interviews. Quality \& Quantity, 36(4), 391-409.

Breslau, J. \& Kendler, K. S. (2005). Lifetime risk and persistence of psychiatric disorders across ethnic groups in the United States. Psychological Medicine, 35 (3), 317-327. http:// doi.org/10.1017/S0033291704003514

Casanueva, C., Martin, S. L., Runyan, D. K., Barth, R. P., \& Bradley, R. H. (2008). Parenting services for mothers involved with child protective services: Do they change maternal parenting and spanking behaviors with young children?. Children and Youth Services Review, 30(8), 861-878.

Cavazos-Rehg, P. A., Zayas, L. H., \& Spitznagel, E. L. (2007). Legal status, emotional well-being and subjective health status of Latino immigrants. Journal of the National Medical Association, 99(10), 1126-1131.

Cunradi, C. B., Caetano, R., \& Schafer, J. (2002). Socioeconomic predictors of intimate partner violence among White, Black, and Latino couples in the United States. Journal of Family Violence, 17(1), 377-389.

Da-Silva, V. A., Morales-Santos, A. R., Carvalho, M .S., Martins, M. L. P., \& Teixeira, N. A. (1998). Prenatal and postnatal depression among low income Brazilian women. Brazilian Journal of Medical and Biological Research, 31 (6), 799-804.

Dutton, M. A., Green, B. L., Kaltman, S. I., Roesch, D. M., Zeffiro, T. A., \& Krause, E. D. (2006). Intimate partner violence, PTSD, and adverse health outcomes. Journal of Interpersonal Violence, 21(7), 955-968. http://doi.org/ $10.1177 / 0886260506289178$

Edelson, M., Hokoda, A., \& Ramos-Lira, L. (2007). Differences in effects of domestic violence between Latina and non-Latina women. Journal of Family Violence, 22(1), 1-10. Doi: http://doi. org/10.1007/s10896-006-9051-1

Enríquez, J. F., González, F. E., Manríquez, P. A., Rivera, A, E., \& Pérez, M. C., \& Mendoza, M. 
(2010). Prevalencia de depresión en usuarios de los servicios ambulatorios de salud en el estado de Guanajuato. Pensamiento Psicológico, 7(1), 53-62.

Esteinou, R. (2005). Parenting in Mexican Society. Marriage \& Family Review, 36 (4), 7-29. Doi: http://doi.org/10.1111/J1741-3729.2000.00025.x

Flake, D. F. \& Forste, R. (2006). Fighting families: Family characteristics associated with domestic violence in five Latin American countries. Journal of Family Violence, 21(1), 19-29. Doi: http://doi.org/10.1007/s10896-005-9002-2

García de Alba, J. E., Castañeda, E., Pando, M., \& Aranda, C. (2011). Depresión en asistentes médicas: Análisis de los factores de riesgo sociolaborales. Psicología y Salud, 21(1), 73-78.

Glaser, B. G. (1965). The constant comparative method of qualitative analysis. Social Problems, 12(4), 436-445. Doi: http://doi.org/10.1525/ sp.1965.12.4.03a00070

Gustafsson, H. C., Cox, M. J., \& Blair, C., (2012). Maternal parenting as a mediator of the relationship between intimate partner violence and effortful control. Journal of Family Psychology, 26(1), 115-123. Doi: http://doi.org/10.1037/a0026283

Harris, R. J., Firestone, J. M., \& Vega, W. A. (2005). The interaction of country of origin, acculturation, and gender role ideology on wife abuse. Social Science Quarterly, 86 (2), 463-481. Doi: http:// doi.org/10.1111/j.0038-4941.2005.00313.x

Hovey, J. D. (2000). Acculturative stress, depression, and suicidal ideation in Mexican Immigrants. Cultural Diversity and Ethnic Minority Psychology, 6(2), 134-151. Doi: http://doi.org/10.1037/1099-9809.6.2.134

Johnson, M. P. (2008). A typology of domestic violence. Boston: Northeastern University Press.

Krishnakumar, A. \& Buehler, C. (2000). Interparental conflict and parenting behaviors: A meta-analytic review. Family
Relations, 49(1), 25-44. Doi: http://doi. org/10.1111/j.1741-3729.2000.00025.x

Masten, W. G., Asidao, C. S., Jerome, W. W., Mosby, L., Caldwell-Colbert, A. T., ... Ervin, S. (2004). Depression and acculturation in Mexican American and European American women. Anales de Psicología, 20(1), 15-21.

Morales-Carmona, F., Luque-Coqui, M., \& BarrosoAguirre, J. (2002). Alteraciones emocionales en una muestra de mujeres mexicanas con eventos ginecoobstétricos. Perinatología y Reproducción Humana, 16(4), 157-162.

Organización de las Naciones Unidas (2018). Informe anual 2017-2018 de ONU mujeres. New York: ONU mujeres.

Orozco, A. E. (2016). Violencia de pareja más allá de la frontera: La pesadilla continúa ahora en Estados Unidos. Revista Intercontinental de psicología y educación, 18(1-2), 137-156.

Ortega, L., Lartigue, T., \& Figueroa, M. E. (2001). Prevalencia de depresión, a través de la escala de depresión perinatal de Edinburgh (EPDS), en una muestra de mujeres mexicanas embarazadas. Perinatología y Reproducción Humana, 15(1), 11-20.

Parra-Cardona, J. R., Bulock, L. A., Imig, D. R., Villaruel, F. A., \& Gold, S. J. (2006). Trabajando duro todos los días: Learning from the experiences of Mexican-origin migrant families. Family Relations, 55(1), 361-375.

Parrado, E. A. \& Flippen, C. A. (2005). Migration and gender among Mexican women. American Sociological Review, 70 (4), 606-632. Doi: http://doi.org/10.1177/000312240507000404

Rosenbaum, A. \& Leisring, P. A. (2003). Beyond power and control: towards an understanding of partner abusive men. Journal of Comparative Family Studies, 34(1), 7-23.

Sullivan, M. M. \& Rehm, R. (2005). Mental health of undocumented Mexican immigrants: A review of the literature. Advances in Nursing 
Sciences, 28(3), 240-251. Doi: http://doi. org/10.1097/00012272-200507000-00006

Tafarodi, R. W. \& Swann, W. B. (1995). Self-liking and self-competence as dimensions of global selfesteem: Initial validation of a measure. Journal of Personality Assessment, 65(2), 322-342.

Terrazas-Carrillo, E. C. \& McWhirter, P. T. (2015). Employment status and intimate partner violence among Mexican women. Journal of interpersonal violence, 30(7), 1128-1152.
Venguer, T., Fawcett, G., Vernon, R., \& Pick, S. (1998). Violencia doméstica: Un marco conceptual para la capacitación del personal de salud. New York: Population Council.

Weaver, C., Shaw, D., Dishion, T., \& Wilson, M. (2008). Parenting self-efficacy and problem behavior in children at high risk for early conduct problems: The mediating role of maternal depression. Infant Behavior \& Development, 31(4), 594-605. Doi: http://doi.org/10.1016/j. infbeh.2008.07.006 